\title{
SARS-CoV-2 infection in hemodialysis patients: Preliminary data from a Portuguese hospital center
} \author{
António Cabrita ${ }^{1}$, Rui Sarmento e Castro ${ }^{3}$ \\ ${ }^{1}$ Serviço de Nefrologia, Centro Hospitalar Universitário do Porto \\ 2 Serviço de Medicina Interna, Centro Hospitalar Universitário do Porto \\ ${ }^{3}$ Serviço de Infeciologia, Centro Hospitalar Universitário do Porto
}

Joana Tavares ${ }^{1}$, Bruno Fraga Dias ${ }^{1}$, João Pedro Oliveira ${ }^{1}$, Inês Sala ${ }^{1}$, Filipa Silva ${ }^{1}$, Ana Castro ${ }^{1}$, Cristina Freitas $^{1}$, Tomás Fonseca ${ }^{2}$, João Pedro Pimentel ${ }^{1}$

\section{ABSTRACT}

Background: In December 2019, a new form of coronavirus was identified (COVID-19) and quickly became a worldwide pandemic. Previous data reported a more severe disease in older patients, with high comorbidities, which are common features in hemodialysis patients. Scarce data is available about the clinical presentation and outcomes in this population. For this reason, we aim to report the characteristics of COVID-19 disease in hemodialysis patients admitted to our center. Methods: We retrospectively reviewed all patients on chronic hemodialysis admitted with a laboratory-confirmed COVID-19 infection from March $2^{\text {nd }}$ to May $20^{\text {th }}$ of 2020 . Baseline characteristics, clinical, laboratory and radiological presentation were registered and treatment and outcomes analysed. Results: Fourteen patients were included, $57 \%$ female, with a median age of 81 years old and a high number of comorbidities. Cough and fever were the most common symptoms and lymphocytopenia, increased inflammatory parameters and coagulation dysregulation were frequently registered. Ground-glass opacities were present in each thorax computerized tomography performed. Five patients developed respiratory insufficiency, but none needed invasive ventilation or ICU admission. In a significant proportion, discharge was postponed due to the impossibility of ensuring social isolation. Median viral shedding was 38 days. Discussion and conclusion: Hemodialysis patients who required hospitalization were very old and had multiple comorbidities. Even so, the impaired immune response of this population seems to cause a less severe course of the disease and a longer time of viral shedding. HD units have required a reformulation of their circuits in order to avoid disease spreading.

Key words: COVID-19; End-stage renal disease; Hemodialysis; Kidneys; SARS-CoV-2.

\section{INTRODUCTION}

The novel coronavirus disease 2019 (COVID-19), first noted in Wuhan (China), has quickly spread worldwide and as of 11 March 2020 was considered a global pandemic by the World Health Organization $^{1}$.

Hemodialysis (HD) patients represent a specific population at risk for the current outbreak of severe acute respiratory syndrome coronavirus (SARS-CoV-2). These patients are at higher risk due to factors related to their advanced age, coexistent comorbidities and permanent state of relative immunosuppression, plus because of the need for frequent visits ( 3 times a week) to HD units and gathering with other patients and health-care providers ${ }^{2}$.

The control of COVID-19 in dialysis units represents a challenge and for this reason, the Portuguese Health Ministry along with the National Dialysis Monitoring Commission (CNAD-Comissão Nacional de Acompanhamento de Diálise) drew up specific guidelines for this group of patients ${ }^{3}$.

In May $20^{\text {th }}$ of 2020 , there were $4,789,205$ confirmed cases and 318,789 deaths worldwide; in Portugal there were 29660 confirmed cases, 1263 deaths, 609 admitted to hospital and 93 in the ICU4,5.
To date, scarce data is available concerning clinical and analytical characteristics of SARS-Cov-2 infection in chronic hemodialysis (HD) patients. Early reports suggest a less severe presentation in the light of the immunosuppressed state of this population ${ }^{6}$.

In this study, we report COVID-19 disease characteristics in regular HD patients that were admitted to our hospital with COVID-19.

\section{METHODS}

\section{Study Design}

This is a retrospective study, performed on patients admitted to our center who met the criteria below. Clinical data were reviewed, and demographic, clinical, analytical and patient outcomes assessed.

Defined as a case were all patients on chronic hemodialysis, admitted to our center due to COVID-19 disease, with a laboratory confirmation of SARS-CoV2 infection through identification of viral RNA in the nasopharynx or oropharynx by real-time reverse transcription polymerase chain reaction (RT-PCR) technique ${ }^{7}$. 
Included were patients hospitalized due to the need for differentiated medical care or inability to maintain in isolation at home ${ }^{3}$. Patients that had initiated kidney replacement therapy, patients in peritoneal dialysis and kidney transplant patients were excluded. Patients with COVID-19 admitted for other reasons, not related to the infectious disease, were also not considered.

Patients were selected from among the cases admitted between March $2^{\text {nd }}$ and May 20th 2020.

\section{Microbial sample collection and analysis}

Tests were performed on patients suspected of having the disease either due to the presence of symptoms or by having been in close contact with confirmed patients. The laboratory diagnosis of SARS-CoV-2 infection was carried out through nucleic acid amplification tests (NAAT) that detect unique sequences of the virus, using RT-PCR. At this point, we used Liferiver Novel Coronavirus (SARS-CoV-2) real time multiplex RT-PCR kit (Liferiver/Shanghai ZJ Bio-Tech Co.), which is a CE-IVD marked test for the simultaneous qualitative detection of 3 SARS-CoV-2 target viral genes (gene $\mathrm{E}$, gene $\mathrm{N}$ and gene ORF1ab). Laboratory procedures were strictly performed according to the manufacturers' instructions.

\section{Clinical management}

All patients admitted to our center with confirmed disease were allocated to an isolation unit created for that purpose, with the necessary equipment to perform conventional hemodialysis, avoiding intra-hospital dislocations and, consequently, reducing the risk of in-hospital transmission.

Almost all patients included in the study underwent blood analysis and chest imaging following a protocol established at the hospital; some patients, for reasons related to clinical stability, did not undergo imaging.

During the hospitalization period, patients maintained regular conventional intermittent hemodialysis.

Patients were treated by a multidisciplinary team (nephrology, internal medicine and infectious diseases) following the updated scientific recommendations.

Regarding the treatment and according to our hospital protocol, patients with documented pneumonia or respiratory failure started hydroxychloroquine $200 \mathrm{mg}$ every other day. In all patients, an electrocardiogram was performed to access corrected QT interval before the administration of the drug.

\section{Follow-up}

The patients were followed up during the hospitalization period and after discharge until they presented criteria for cure or until death. The tests to assess cure, if positive, were repeated within 7 days. Data were collected until May $20^{\text {th }}$ or until death.
Patients showed healing criteria after at least 7 days of symptom resolution and two negative RT-PRC SARS-CoV2 tests, separated by 24-48 hours.

\section{Statistical analysis}

All statistical analyses were performed using Microsoft Excel 2016 (Microsoft ${ }^{\mathrm{TM}}$ ). Continuous variables were expressed as median (interquartile range - IQR) and categorical variables were presented as frequency (percentage - \%).

\section{RESULTS}

In this period, 17 dialysis patients had COVID-19 disease, but 3 patients were excluded: one admitted with uremic syndrome at dialysis start and that contracted the infection during hospital stay; a patient that transitioned to peritoneal dialysis due to refractory central venous catheter infection; and a patient with COVID-19 followed in ambulatory but admitted due to a vascular access problem. Fourteen patients met the inclusion criteria of our study.

\section{Baseline characteristics}

Our population had a median age of 81 (76-83) years old; the youngest patient was 64 and the oldest 91 years old. Patients had an important degree of functional dependence, with $72 \%$ of the patients with ECOG equal to or greater than 2. Eight patients (57\%) were female.

Several co-morbidities were reported, with emphasis on arterial hypertension, heart failure and type 2 diabetes mellitus, respectively $86 \%, 79 \%$ and $71 \%$. Despite the high prevalence of hypertension, only $14 \%$ of patients were taking ACE inhibitors at admission.

Diabetic kidney disease was the main etiology of chronic kidney disease (CKD), present in half of the patients. The median time of renal replacement therapy was 3.1 (1.9-9.3) years and arteriovenous fistula was the vascular access present in most of the patients (86\%). The rest of the patients had a central venous catheter.

\section{Clinical presentation, laboratory and radiology findings}

Table I and Table II summarize clinical manifestations and laboratory findings, respectively.

The median interval between the onset of symptoms and the first positive test was $2(0.15-3)$ days.

The main symptom was dry cough (64\%), followed by fever (36\%), dyspnoea and fatigue (29\%). Only 1 patient had diarrhea and no patient registered any other gastrointestinal manifestations, namely abdominal pain or vomiting. No patient was completely asymptomatic.

At the time of admission, most patients (79\%) had leukopenia (defined by leukocyte count less than 1500) and $50 \%$ of patients had 


\section{Table 1}

Symptoms presented at admission ( $\mathrm{n}$ - number of patients, \%)

\begin{tabular}{l||c|c|}
\multicolumn{1}{c|}{ Symptoms } & $\mathbf{n}$ & $\%$ \\
\hline Dry cough & 9 & 64 \\
Dyspnea & 4 & 29 \\
Fever & 5 & 36 \\
Fatigue & 4 & 29 \\
Myalgia & 2 & 14 \\
Rhinorrhea & 1 & 7 \\
Diarrhea & 1 & 7 \\
\hline
\end{tabular}

\section{Table 2}

Laboratory findings at admission - Median (IQR) and Hospital Reference Values

\begin{tabular}{l|c|c}
\multicolumn{1}{c|}{ Laboratory findings } & Median (IQR) & Reference values \\
\hline White blood cells, $\times 10^{9} / \mathrm{L}$ & $4.3(3.8-5.2)$ & $4.00-11.00$ \\
Lymphocyte count, $\times 10^{9} / \mathrm{L}$ & $1.1(0.7-1.4)$ & $1.50-4.00$ \\
Hemoglobin, $\mathrm{g} / \mathrm{L}$ & $11(10.1-11.9)$ & $13-17$ \\
Platelets $\times 10^{9} / \mathrm{L}$ & $146(105.7-171)$ & $150-450$ \\
Lactate dehydrogenase. $\mathrm{U} / \mathrm{L}$ & $247.5(189-292)$ & $135-225$ \\
Creatinine kinase, $\mathrm{U} / \mathrm{L}$ & $54.4(33.3-137)$ & $24-204$ \\
\hline Myoglobin $\mathrm{ng} / \mathrm{ml}$ & $374.3(176-679)$ & $28-72$ \\
Albumin, $\mathrm{g} / \mathrm{L}$ & $35.8(34.5-37.1)$ & $35-50$ \\
Serum ferritin, $\mu \mathrm{gg} / \mathrm{L}$ & $1202(1065-1541)$ & $12.8-454$ \\
PCR, $\mathrm{mg} / \mathrm{dL}$ & $39.8(12.4-81.2)$ & $0.0-5.0$ \\
Procalcitonin, $\mathrm{ng} / \mathrm{ml}$ & $0.64(0.35-1.76)$ & $0.00-0.05$ \\
Prothrombin time, s & $11.3(11-12.5)$ & $<11$ \\
Partial thromboplastin time, s & $31(28-36)$ & $<27.6$ \\
D-Dimer, $\mathrm{ng} / \mathrm{ml}$ & $526(304-683)$ & $<300$
\end{tabular}

thrombocytopenia (defined by platelets count less than 150000). Increased LDH and inflammatory markers, including CRP, procalcitonin and ferritin levels was observed. Plus, high prothrombin and partial thromboplastin time were registered.
Four patients' thorax x-rays revealed no significant changes. Three patients performed thorax computerized topographies and the most common finding was a pattern of ground-glass opacity.

\section{Treatment and outcomes}

All patients were submitted to low-flux HD.

According to hospital protocol, four patients underwent hydroxychloroquine therapy for 6 days (4.75-6) and no adverse effects were reported. No antiviral therapy was performed.

Throughout hospital stay, antibiotics for concurrent respiratory bacterial infection were started in five patients. Ten days of beta-lactams combined with three days of azithromycin was the most used therapy. However, in only one of them, a microbiological agent (Klebsiella pneumoniae) was isolated. Three patients showed evidence of bacterial infection of non-respiratory origin: two urinary infections and one parotitis, treated, respectively, with beta-lactam for 10 days, fosfomycin for 1 day and clindamycin for 10 days.

Five patients (36\%) developed respiratory insufficiency during the follow-up period; type 1 was registered in 3 patients and type 2 in two. It took a median of 16 (10-22.5) days for complete resolution. Particularly, one patient developed severe respiratory insufficiency for 23 days, requiring a high oxygen support, a long spectrum empirical antibiotic regimen and a single dose of methylprednisolone (125mg). In this case, chest CT documented diffuse involvement by COVID 19 and aspects suggestive of bacterial infection (Figure 1).

Another patient developed global respiratory failure and therefore needed non-invasive ventilation (NIV) for two periods, with good clinical response. No patient progressed to acute respiratory distress syndrome (ARDS), with the need of invasive ventilation and intensive care unit (ICU) stay.

During the follow-up period, there were two deaths, representing a mortality rate of $14 \%$. One patient died during hospital stay and the other 15 days after discharge; both patients were older than 80 years

\section{Figure 1}

Computerized tomography thorax scans of patient with COVID-19 and concurrent bacterial infection.
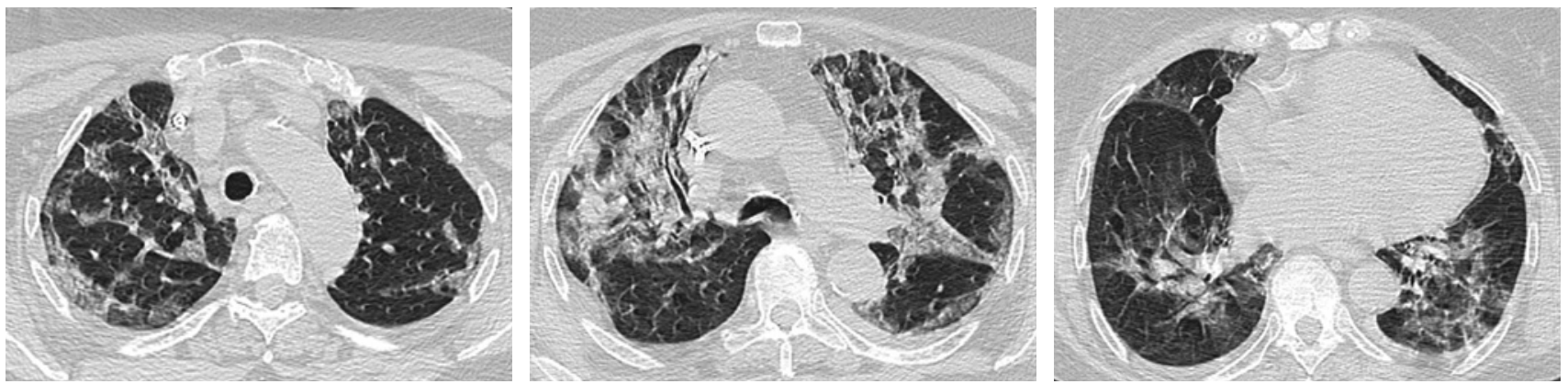
old and had a high number of comorbidities. In the later, no evident cause of death was identified.

The length of stay was prolonged in a great percentage $(72 \%)$ of patients due to the inability to maintain in isolation at home.

The average duration of viral shedding was 38 (33-50) days and the longest was 65 days. At the time of writing this paper, all living patients accomplished the criteria for cure.

\section{DISCUSSION}

Chronic hemodialysis patients with COVID-19 were more likely to be of older age and have more comorbidities, which would make them a group at higher risk. Plus, patients undergoing hemodialysis are also believed to have an impaired immune response, being at higher risk of infectious disease, higher complications and increased mortality rates ${ }^{8}$.

However, as was the case with SARS-CoV infection, it is thought that the severity of SARS-CoV-2 infection is caused not only by the direct toxicity of the virus but also by a hyperactive and dysregulated immune response ${ }^{9}$. This would mean that the relative immunosuppressed basal state of patients under dialysis could lead to a milder disease presentation. Additionally, Pisani A. et al reported a protective role of heparin, a common anticoagulant used during $\mathrm{HD}$, in preventing the pseudo virus entry into the host cells ${ }^{10}$.

Accordingly, early Chinese reports from HD units suggested a less severe respiratory picture in these patients ${ }^{6}$ and more recent data from Italy also support this initial information ${ }^{11}$.

In fact, the disease symptoms of our patients were similar to the general Portuguese population in which cough (41\%) followed by fever (29\%) were the most common registered symptoms ${ }^{4}$. The largest report of patients so far from Wuhan described almost a quarter of patients as asymptomatic ${ }^{12}$. Although we had no asymptomatic patients, this can represent a bias, since there was no standard screening in all Portuguese HD units.

While analysing the overall Portuguese mortality rate of $4 \%$, one could think that the mortality rate in this subpopulation was significantly higher (14\%). But analyzing the mortality rate of patients from the same age group (over 70 years old), that at May $20^{\text {th }}$ was approximately $16 \%$ (1096 deaths in a total of 6810 patients), we can observe that the numbers are not different ${ }^{4}$.

Similarly to the Wuhan reports ${ }^{12}$, lymphocytopenia was a common finding. Still, there is lack of evidence whether it is related to a more severe form of the disease ${ }^{12,13}$. The elevated inflammation markers also support the hyperactive immune response underlying the SARS-CoV-2 infection ${ }^{9}$.

Significant changes regarding coagulation have also been registered, as coagulation seems to represent another line of defence against infections ${ }^{14}$. In addition to increased prothrombin and partial activated thromboplastin times, thrombocytopenia was also frequent in our patients. A Chinese meta-analysis suggested a relationship between thrombocytopenia and a more severe COVID-19 infection ${ }^{15}$, but these results were not reproducible in other studies ${ }^{12,13}$.

In the few CT scans preformed, we could verify, in all patients, the presence of the typical ground-glass opacities that are associated to the SARS-COV-2 infection ${ }^{16}$. Gattioni et. al, described two phenotypes of COVID-19 pneumonia, the above-mentioned lesions as the phenotype $\mathrm{L}$, and the phenotype $\mathrm{H}$ as a kind of severe $\mathrm{ARDS}^{17}$. The latter wasn't verified in our population. In fact, even our patient with the worst respiratory presentation, with type 2 IR and need for NIV, exhibited a type $L$ phenotype.

Current recommendations suggest modality choice should be according the logistic and experience of each center ${ }^{18}$. In our center, a dedicated COVID-19 HD area was created from a pre-existing inward room, far from our regular HD area and from the water treatment room. Low-influx HD was performed due to the lack of access to ultrapure water.

Our therapeutic choices should also be reviewed. Although promising in vitro reports have shown that both chloroquine and hydroxychloroquine, particularly the latter, have a potent antiviral activity ${ }^{19}$, clinical trials have failed to show their efficacy ${ }^{20,21}$. Even so, the use of the well-known hydroxychloroquine was revealed to be attractive as it was associated to a higher proportion of patients with a negative molecular result three days after its start ${ }^{22}$. Systemic glucocorticoid use is not recommended, unless there is a clear indication for its administration, such as the exacerbation of a chronic obstructive pulmonary disease 23 .

According to Portuguese Health Ministry recommendations, every patient on dialysis with COVID-19 had to have an initial evaluation at the hospital ${ }^{24}$. Only after that, and if the patient was clinically stable, could discharge be considered. For this reason, HD units had to reorganize their logistics, creating dedicated shifts, with dedicated staff, for patients with COVID-19 disease.

Even after hospital discharge, it was possible to access patient information about viral shedding. In an early multicenter cohort retrospective study from Wuhan of almost 200 patients, 37 days was reported as the longest viral shedding 25 . A more recent paper gives a longer time of viral shedding in critically ill patients, the longest 52 days. The extended time seemed to be related to an immunity impairment of this group of patients ${ }^{26}$. Reinforcing again the idea of the relative state of immunosuppression of patients under dialysis, one can easily understand the long viral shedding time, median 38 days and longest 65 days, registered in our population.

Bearing in mind that HD units could represent a high risk of cross-contamination between patients and health providers, and in the light of CNAD recommendations ${ }^{3}$, the units quickly created their own contingency plans.

Every step of the dialytic treatment was rethought. A pre-admission triage was performed, either by nurses or doctors, to guarantee that no patient with possible symptoms of COVID-19 reached the unit and to forward these patients to the dedicated areas; measures were also 
taken concerning patient transport, with a limited number of patients per ambulance, plus the use of their own means of transportation was encouraged in patients for whom this was possible; the body temperature of patients and health providers was registered before admission to the unit and a surgical mask was provided for each treatment; food and medication were given to the patients to take at their homes.

\section{CONCLUSION}

In summary, even though it represents a small population, this Portuguese study provides information about patients under a regular program of hemodialysis.

Against all odds, this group of patients, appeared to have a less severe course of the disease than the same age group of the general population. In addition, clinical and laboratorial presentation seem also similar. On the other hand, time of viral shedding seems longer. These features could be a result of the relative immunosuppressed state of patients under dialysis.

Further, patients on hemodialysis represented a challenge in terms of logistics, not only in the creation of specific areas in the hospitals, but also in the recreation of the HD units' circuits.

We eagerly wait for the results of a larger sample of patients, such as the first European report directed by EDTA on HD and transplant patients infected with COVID-19 (ERACODA COVID-19 KRT), to reach further conclusions.

Disclosure of potential conflicts of interest: none declared

\section{References}

1. Organization WH. Coronavirus disease (COVID-19) Pandemic [updated 2020, June 8th Available from: https://www.who.int/emergencies/diseases/novel-coronavirus-2019.

2. Weiner DE, Watnick SG. Hemodialysis and COVID-19: an Achilles' heel in the pandemic health care response in the United States. Kidney Med. 2020;2(3):227-230.

3. Saúde DGd. COVID-19: FASE DE MITIGAÇÃO - Doentes com Doença Renal Crónica em Hemodiálise [updated 2020, May 23. Available from: https://www.dgs.pt/directrizes-da-dgs/normas-e-circulares-normativas/norma-n-0082020-de-280320201.aspx.

4. Saúde DGd. COVID-19 - Relatório de Situação a 20/05/2020 [updated 2020, May 20th. Available from: https://covid19.min-saude.pt/wp-content/uploads/2020/05/79 DGS boletim 20200520.pdf.

5. Organization WH. Coronavirus disease (COVD-19) Situation Report - 121 [updated 2020, May 20th. Available from: https://www.who.int/docs/default-source/coronaviruse/situation-reports/20200520-covid-19-sitrep-121.pdf?sfvrsn=c4be2ec6 4 .
6. Ma Y, Diao B, Lv X, Zhu J, Liang W, Liu L, et al. 2019 Novel coronavirus disease in hemodialysis (HD) patients: report from one HD center in Wuhan, China. medRxiv. 2020:2020.02.24.20027201.

7. Chan JF, Yip CC, To KK, Tang TH, Wong SC, Leung KH, et al. Improved molecular diagnosis of COVID-19 by the novel, highly sensitive and specific COVID-19-RdRp/Hel real-time reverse transcription-PCR assay validated In vitro and with clinical specimens. J Clin Microbiol. 2020;58(5):e00310-20.

8. Betjes MG. Immune cell dysfunction and inflammation in end-stage renal disease. Nat Rev Nephrol. 2013;9(5):255-265.

9. Xu Z, Shi L, Wang Y, Zhang J, Huang L, Zhang C, et al. Pathological findings of COVID-19 associated with acute respiratory distress syndrome. Lancet Respir Med. 2020;8(4):420-422.

10. Pisani A, Rizzo M, Angelucci V, Riccio E. COVID-19 Experience in hemodialysis patients: a cue for therapeutic heparin-based strategies? Nephron. 2020;144(8):383-385.

11. Alberici F, Del Barba E, Manenti C, Econimo L, Valerio F, Pola A, et al. Managing patients in dialysis and with kidney transplant infected with Covid-19. G Ital Nefrol. 2020;37(2):2020-vol2.

12. Xiong F, Tang H, Liu L, Tu C, Tian J-B, Lei C-T, et al. Clinical characteristics of and medical interventions for COVID-19 in hemodialysis patients in Wuhan, China. J Am Soc Nephrol. 2020;31(7):1387-1397.

13. Lagadinou M, Salomou EE, Zareifopoulos N, Marangos M, Gogos C, Velissaris D. Prognosis of COVID-19: changes in laboratory parameters. Infez Med. 2020;28(1):89-95.

14. Han H, Yang L, Liu R, Liu F, Wu K-I, Li J, et al. Prominent changes in blood coagulation of patients with SARS-CoV-2 infection. 2020(0):20200188.

15. Lippi G, Plebani M, Henry BM. Thrombocytopenia is associated with severe coronavirus disease 2019 (COVID-19) infections: A meta-analysis. Clin Chim Acta. 2020;506:145-148.

16. Wang $Y$, Dong C, Hu Y, Li C, Ren Q, Zhang X, et al. Temporal changes of CT findings in 90 patients with COVID-19 pneumonia: a longitudinal study. Radiology.0(0):200843.

17. Gattinoni L, Chiumello D, Caironi P, Busana M, Romitti F, Brazzi L, et al. COVID-19 pneumonia: different respiratory treatments for different phenotypes? Intensive Care Medicine. 2020;46(6):1099-1102.

18. Alberici F, Delbarba E, Manenti C, Econimo L, Valerio F, Pola A, et al. Management of patients on dialysis And with kidney transplant during SARS-COV-2 (COVID-19) pandemic In Brescia, Italy. Kidney Int Rep. 2020;5(5):580-585.

19. Yao X, Ye F, Zhang M, Cui C, Huang B, Niu P, et al. In vitro antiviral activity and projection of optimized dosing design of hydroxychloroquine for the treatment of severe acute respiratory syndrome Coronavirus 2 (SARS-CoV-2). Clin Infect Dis. 2020;71(15):732-739.

20. Investigatiors Rt. No clinical benefit from use of hydroxychloroquine in hospitalised patients with COVID-19 [Available from: https://www.recoverytrial.net/files/hcq-recovery-statement-050620-final-002.pdf.

21. Tang W, Cao Z, Han M, Wang Z, Chen J, Sun W, et al. Hydroxychloroquine in patients with mainly mild to moderate coronavirus disease 2019: open label, randomised controlled trial. BMJ. 2020;369:m1849.

22. Gautret $P$, Lagier JC, Parola P, Hoang VT, Meddeb L, Mailhe M, et al. Hydroxychloroquine and azithromycin as a treatment of COVID-19: results of an open-label non-randomized clinical trial. Int J Antimicrob Agents. 2020:105949.

23. (CDC) CfDCaP. Interim Clinical Guidance for Management of Patients with Confirmed Coronavirus Disease (COVID-19) [updated June 2, 2020. Available from: https://www.cdc.gov/coronavirus/2019-ncov/hcp/clinical-guidance-management-patients.html.

24. Saúde DGd. COVID-19: FASE DE MITIGAÇÃO - Abordagem do Doente com Suspeita ou Infeção por SARS-CoV-2 [updated April 25, 2020. Available from: https://www.dgs.pt/directrizes-da-dgs/ normas-e-circulares-normativas/norma-n-0042020-de-23032020-pdf.aspx.

25. Zhou F, Yu T, Du R, Fan G, Liu Y, Liu Z, et al. Clinical course and risk factors for mortality of adult inpatients with COVID-19 in Wuhan, China: a retrospective cohort study. Lancet. 2020;395(10229):1054-1062

26. Chen J, Lu H, Melino G, Boccia S, Piacentini M, Ricciardi W, et al. CoVID-19 infection: the China and Italy perspectives. Cell Death Dis. 2020;11(6):438.

\section{Correspondence to:}

Joana Tavares, MD

Serviço de Nefrologia, Centro Hospitalar Universitário do Porto

Largo Prof. Abel Salazar 4099-001 Porto, Portugal

E-mail: joanatavares91@gmail.com 